IN-SITU X-RAY DIFFRACTION STUDIES OF IRON ORE SINTER PHASES

N. V. Y. Scarlett ${ }^{1}$ M. I. Pownceby ${ }^{1}$ A. N. Christensen ${ }^{2}$ I. C. Madsen ${ }^{1}$ ${ }^{1}$ CSIRO Minerals Box 312 CLAYTON SOUTH VICTORIA 3169

AUSTRALIA ${ }^{2}$ Aarhus University, Department of Chemistry, Aarhus University Langelandsgade 140 DK-8000 Aarhus C, Denmark

Due to the depletion of world lump iron ore stocks, pre-treated agglomerates of fine ores are making up a growing proportion of blast furnace feedstock (around $80 \%$ ). These agglomerations, or 'sinters', have the potential to be quite variable in their composition. However, low temperature sintering $\left(<1300^{\circ} \mathrm{C}\right)$ of many hematite-goethite ores, fluxed to a fixed basicity, produces sinters that are reasonably consistent in their mineralogy. Such sinters are generally composed of four main phases: iron oxides $(40-70 \mathrm{vol} \%)$, ferrites (most of which are SFCA, Silico-Ferrite of Calcium and Aluminium, 20-50vol\%), glasses (up to 10vol\%) and dicalcium silicates (up to 10vol\%). Despite the high proportion of SFCA and the importance of its role in sinter, it is yet to be adequately characterized in terms of its chemistry and mineralogy. To date, there has been much work published regarding the fundamental phase relations, which influence the assemblages and textural features normally encountered in iron ore sinters.

However, these works report the results of equilibrium studies, and, whilst important in establishing fundamental platforms for the study of sintering, they are unlikely to reflect conditions operating during industrial sintering processes. Real-time, X-ray diffraction (XRD) experiments have been conducted into the formation of SFC (aluminium-free homologue of SFCA). This allows the study of the mechanism of SFC formation and the observation of intermediate phases directly with respect to time and temperature. Application of the Rietveld method for phase quantification also allows the estimation of kinetic data for the mineral phases being studied.

Keywords: (IN SITU) (HIGH TEMPERATURE) (IRON ORE SINTERING)

Acta Cryst. (2002). A58 (Supplement), C363

\section{LOW TEMPERATURE CHARGE ORDERING IN $\mathrm{Fe}_{3} \mathrm{O}_{2} \mathrm{BO}_{3}$} LUDWIGITE

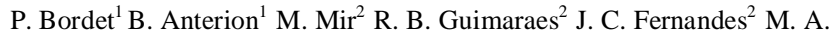
Continentino ${ }^{2}$

${ }^{1}$ Laboratoire De Cristallographie CNRS BP 166 GRENOBLE CEDEX 9 38042 FRANCE ${ }^{2}$ Instituto de Fisica, Universidade Federal Fluminense, Campus da Praia Vermelha, Niteroi, 24.210-340, RJ, Brazil

The structure (space group Pbam, a = 9.462 $\AA, \mathrm{b}=12.308 \AA, \mathrm{c}=3.075 \AA$ ) of the ludwigite $\left(\mathrm{Fe}^{2+}\right)_{2}\left(\mathrm{Fe}^{3+}\right) \mathrm{O}_{2} \mathrm{BO}_{3}$ oxyborate is made of zigzag walls of edgesharing $\mathrm{FeO}_{6}$ octahedra, connected by $\mathrm{BO}_{3}$ triangular groups. It contains subunits arranged as three leg ladders of $\mathrm{Fe}$ cations in intermediate valence state, with one electron delocalized among the three $\mathrm{Fe}^{3+}$ sites forming the ladder rungs. The low dimensionality and presence of different sublattices for $\mathrm{Fe}$ cations with different valences, confer to ludwigite quite unusual properties : physical measurements reveal the existence of three transitions at $112 \mathrm{~K}, 70 \mathrm{~K}$ and $40 \mathrm{~K}$.

Anomalies in magnetization and resistivity curves are also observed close to room temperature. Recently (M. Mir et al., Phys. Rev. Lett. 87-14, (2001), 147201), was reported the appearance of a superstructure at $283 \mathrm{~K}$, with doubling of the c-axis, attributed to electron localization and $\mathrm{Fe}-\mathrm{Fe}$ pair formation. Structural data obtained at $294 \mathrm{~K}$ and $140 \mathrm{~K}$ were presented. However, the $294 \mathrm{~K}$ data, very close to this broad transition, may not give an accurate description of the high temperature phase.

To better understand this transition, we have followed the evolution of the structural parameters in an extended temperature range, by collecting x-ray single crystal diffraction data at small temperature intervals, using a NoniusKappaCCD diffractometer with low and high temperature attachments. The results indicate that complete charge ordering is not reached in the low temperature phase, which implies that other structural phase transitions may exist at lower temperatures. Diffraction studies are under way to check this hypothesis.
Acta Cryst. (2002). A58 (Supplement), C363

PHASE TRANSITIONS IN $\left(\mathrm{CsPbCl}_{3}\right)_{1-\mathrm{x}}\left(\mathrm{CsPbBr}_{3}\right)_{\mathrm{x}}$ MIXED CRYSTAL

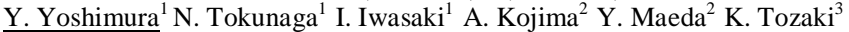
Ritsumeikan University Physics Dept. 1-1-1 Noji-Higashi KUSATU-SHI SHIGA J-525-8577 JAPAN

${ }^{1}$ Faculty of Science and Engineering, Ritsumeikan University ${ }^{2}$ Department of Materials Science, The University of Shiga Prefecture ${ }^{3}$ Department of Physics, Faculty of Education, Chiba University

Phase transitions of the title compound [1], were studied by single crystal Xray diffraction and calorimetric methods. Previously we have reported that the $\mathrm{CsPbCl}_{3}$ crystal displays a very interesting structural phase transitions [1]. As the summarized; (1) the $320 \mathrm{~K}$ transition in $\mathrm{CsPbCl}_{3}$ is a "multi-step transition" within $0.1 \mathrm{~K}$ [2]. (2) The crystal undergoes dual cascade transition starting from the multi-step transition. One is a $320-315-310 \mathrm{~K}$ transition series related to the so-called soft phonon condensation theory. The other is a $320-265-200 \mathrm{~K}$ series having different factors on a transition mechanism, dominating a structural change in the crystal. The results of the X-ray and calorimetric studies of the mixed crystal have shown that the crystal undergoes phase transitions at 314,270 and $210 \mathrm{~K}$. At room temperature, $\mathrm{h}+1 / 2 \mathrm{k}+1 / 21$ type superlattice reflections were observed. The reflections vanish above $314 \mathrm{~K}$. h k $1+1 / 2$ and $\mathrm{h}+1 / 2 \mathrm{k}+1 / 2 \mathrm{l}+1 / 2$ types superlattice reflections were appeared simultaneously below $270 \mathrm{~K}$. The diffraction pattern observed in the hk0 reciprocal plane split along the 010 reciprocal direction below $210 \mathrm{~K}$. The multi-step transition in $\mathrm{CsPbCl}_{3}$ at $320 \mathrm{~K}$ is changed into a single-step transition in the mixed crystal. Transitions corresponding to the 315 and $310 \mathrm{~K}$ in $\mathrm{CsPbCl}_{3}$ were hardly observed in the mixed crystals. The results obtained strongly support the existence of the dominant transition series in the $\mathrm{CsPCl}_{3}$ mentioned above.

References

1: Y.Yoshimura, et al., The 18th IUCr, Glasgow, Scotland, U.K., 1999, P558 2: K.Tozaki at al., Phys. Lett. A, Vol.263, 1999, 203

\section{Keywords: (CSPBCL3)1-X(CSPBBR3)X MIXED CRYSTAL X-RAY DIFFRACTION AND CALORIMETRIC STUDIES CASCADE PHASE TRANSITION}

\section{Acta Cryst. (2002). A58 (Supplement), C363}

PULSED-NEUTRON INVESTIGATIONS OF FIELD-INDUCED TWODIMENSIONAL MAGNETIC MODULATIONS OF DyRu $\mathbf{S i}_{2}$ $\underline{\text { S. Kawano }}^{1}$ M. Takahashi ${ }^{2}$ T. Shigeoka ${ }^{3}$ M.J. Bull ${ }^{4}$ N. Iwata

${ }^{1}$ Research Reactor Institute, Kyoto University Kumatori SENNAN OSAKA 590-0494 JAPAN ${ }^{2}$ Institute of Materials Science, University of Tsukuba ${ }^{3}$ Faculty of Science, Yamaguchi University ${ }^{4}$ ISIS Facility, CLRC, Rutherford Appleton Laboratory ${ }^{5}$ Faculty of Science, Yamaguchi University

DyRu $\mathrm{Si}_{2}$ exhibits a multi-step metamagnetic transition at low temperatures below $\mathrm{T}_{\mathrm{t}}=3.5 \mathrm{~K}$, indicating five field-induced magnetic phases, II, I, III, IV, and V. Recently the magnetic modulation of the zero-field phase II has been established to be two-dimensional with $\mathrm{Q}=(2 / 900)$ of a magnetic unit cell of 9ax9axc, with two paramagnetic Dy ions. We have performed pulsed-neutron investigations of $\mathrm{DyRu}_{2} \mathrm{Si}_{2}$ at $1.5 \mathrm{~K}$ well below $\mathrm{T}_{\mathrm{t}}$ under magnetic fields at PRISMA in ISIS from the point of view of the two-dimensional modulation. For phase II at zero-field the obtained magnetic intensity distribution fully agrees with the results of the two-dimensional modulation, while for phase I at $\mathrm{H}=0.5 \mathrm{~T}$ the modulation becomes again one-dimensional with the reduced magnetization $\mathrm{m}=0.111$, with no paramagnetic Dy ion, similar to a modulation of the high temperature phase above $\mathrm{T}_{\mathrm{t}}$ without field. On the other hand, for phase III at $\mathrm{H}=1.2 \mathrm{~T}$ the modulation becomes again twodimensional with no paramagnetic Dy ion, having $\mathrm{m}=0.423$, and for phase IV another two-dimensional modulation with $\mathrm{m}=0.556$. These two-dimensional modulations can be expressed by the fundamental waves of $\mathrm{Q}=(2 / 900)$ and (0 2/9 0) and their higher harmonics.

Keywords: FIELD INDUCED TWO DIMENSIONAL MAGNETIC MODULATION PULSED NEUTRON SCATTERING DYRU2SI2 